\title{
ON PINNED AND COLLARED MEMBRANES
}

\author{
H. P. W. GOTTLIEB
}

School of Science, Griffith University, Nathan, Queensland 4111, Australia

(January 1999)

Number of pages: 10 


\section{INTRODUCTION}

A long time ago, Rayleigh [1, p. 350] noted that "the fixation of the centre of a vibrating circular membrane does not alter the pitch". The observation was based on a consideration of the effect of raising an excitation frequency through the lowest natural frequency of an ideal membrane. At first glance, this phenomenon appears curious. One is tempted to argue along the following lines. For a membrane pinned at its centre, the amplitude is zero at radial variable $r=0$, so the solutions to the governing Helmholtz equation should not involve the Bessel functions of the second kind, $\mathrm{Y}_{\mathrm{n}}(\mathrm{kr})$, since these all become infinite as $\mathrm{r}$ tends to zero. Moreover, $\mathrm{J}_{0}(\mathrm{kr})$ is equal to unity at $\mathrm{r}=0$, so it also should not appear in the solution. Thus the solutions should only contain Bessel functions of the first kind $\mathrm{J}_{\mathrm{n}}(\mathrm{kr})$ for $\mathrm{n} \geq 1$ (for which the values are zero at $\mathrm{r}=0$ ), and so should not be able to reproduce the fundamental frequency of the complete circular membrane which comes from the fundamental mode involving $\mathrm{J}_{0}(\mathrm{kr})$. However, it can be deduced that there is something wrong with this line of argument, because then the fundamental mode would involve $J_{1}(k r) \cos (\theta)$, where $\theta$ is the polar angle, and so would have nodal radii, contradicting the absence of internal nodal curves for a fundamental mode.

This apparent impasse is resolved by a careful consideration of the problem of an annular membrane with small central exclusion and the behaviour of $\mathrm{Y}_{0}$ for small argument in relation to the resulting characteristic equation, as has been analyzed recently by Wang [2]. The pinned membrane corresponds to central core radius tending to zero. In this paper we elaborate on this phenomenon, and also consider the limit of a "collared" annular membrane with free boundary condition on its inner rim.

\section{THE PINNED CIRCULAR MEMBRANE}


The radial part of the solution to the Helmholtz equation in plane polar coordinates resulting from the two-dimensional wave equation for a vibrating membrane takes the form

$$
u_{n}(r)=a_{n} J_{n}(k r)+b_{n} Y_{n}(k r),
$$

where $J_{n}$ and $Y_{n}$ are ordinary Bessel functions of the first and second kinds [3]. The angular number $\mathrm{n}=0,1,2, \ldots$ corresponds to the angular part of the solution of the form $\cos$ or $\sin$ of $n \theta$. In eq.(1), $k=\omega / c$, where $\omega$ is the radian frequency of vibration and $\mathrm{c}$ is the free wave speed. For an annular membrane with fixed rims at $\mathrm{r}=\varepsilon \mathrm{R}$ and $\mathrm{r}=\mathrm{R}$, the boundary conditions yield the characteristic equation to be satisfied by $\mathrm{k}$ (as in [2], but here with unnormalized outer radius) for the fundamental mode (independent of $\theta: \mathrm{n}=0$ )

$$
J_{0}(k R) Y_{0}(\varepsilon k R)-Y_{0}(k R) J_{0}(\varepsilon k R)=0 \quad .
$$

For small inner or core radius, i.e. small $\varepsilon$, an analytical approximation to the fundamental frequency may be found by using Taylor series expansions and the fact that $J_{0}{ }^{\prime}=-J_{1}, Y_{0}{ }^{\prime}=-Y_{1}$, as well as series expansions for the Bessel functions $[3,4]$, to obtain (c.f. [2])

$$
\begin{aligned}
k R & =j_{0,1}+\kappa, \\
\kappa & \approx \frac{\pi}{2} \frac{Y_{0}\left(j_{0,1}\right)}{J_{1}\left(j_{0,1}\right)} \frac{1}{|\ln (\varepsilon)|} \approx \frac{1.542890}{|\ln (\varepsilon)|},
\end{aligned}
$$

where $\mathrm{j}_{0,1}=2.40482556$ is the lowest zero of $\mathrm{J}_{0}$. Thus, as observed explicitly by Wang [2], there is the surprising result

$$
\mathrm{kR} \rightarrow \mathrm{j}_{0,1} \text { as } \varepsilon \rightarrow 0
$$


so the fundamental frequency for a pinned circular membrane equals that of the ordinary complete circular membrane of the same outer radius.

The parameter $\varepsilon$ actually has to be extremely small before the solution to (2) approaches $\mathrm{j}_{0,1}$ closely, because $1 /|\ln (\varepsilon)|$ tends to zero rather slowly as $\varepsilon$ tends to zero. Moreover, good numerical agreement of the approximation $(3 a, b)$ with the exact solution to eq.(2) is achieved only for very small $\varepsilon$. For instance, even for $\varepsilon=10^{-6}$, there is agreement to 3 significant figures only: $k R \approx 2.52$.

To see how the fundamental mode function itself behaves, it is necessary to carry the analysis a little further. From (1) which vanishes on the outer rim $r=k R$ and (2), the ratio of coefficients is

$$
\mathrm{b}_{0} / \mathrm{a}_{0}=-\mathrm{J}_{0}(\mathrm{kR}) / \mathrm{Y}_{0}(\mathrm{kR}) \approx(\pi / 2)(1 /|\ln (\varepsilon)|)
$$

(where this result follows from (3) and the above-mentioned properties of the Bessel functions). Thus the coefficient $b_{0}$ of the singular function $Y_{0}(k r)$ in (1) tends to zero as the core radius $\varepsilon$ tends to zero (the "infinitely small coefficient" mentioned by Rayleigh [1, p.350]). However, the product $\mathrm{b}_{0} \mathrm{Y}_{0}(\varepsilon \mathrm{kR})$ may consequently be shown to tend to the finite limit $-\mathrm{a}_{0}$ as $\varepsilon$ tends to zero, consistent with the fact that the full mode function (1) vanishes on the inner boundary. Furthermore, for radial variable r not infinitesimally near the inner boundary, in eq.(1) $\mathrm{u}_{0} \rightarrow \mathrm{a}_{0} \mathrm{~J}_{0}\left(\mathrm{j}_{0,1} \mathrm{r} / \mathrm{R}\right)$ as $\varepsilon \rightarrow 0$ by (4) and (5), i.e. for nonzero $r$ the fundamental mode function for infinitesimal core tends to the usual fundamental mode function for the complete circle as the annular membrane tends to the pinned case.

To understand better how this limiting behaviour manifests itself, we now consider a point infinitesimally close to, but not actually on, the inner boundary for infinitesimal core, i.e. we let $r=(1+h) \varepsilon R$, with $h>0$. Then it can be shown that 


$$
\mathrm{u}_{0}((1+\mathrm{h}) \varepsilon \mathrm{R}) / \mathrm{a}_{0} \approx[\ln (1+\mathrm{h})] /|\ln (\varepsilon)| \text { as } \varepsilon \rightarrow 0 .
$$

This shows mathematically how the fundamental mode function (1) evaluated near the inner boundary tends to zero near the infinitesimal core.

The slope $\mathrm{du}_{0} / \mathrm{dr}$ near the inner core may be evaluated from eq.(1) and other properties and is found to be

$$
\left.\frac{d u_{0}}{d r}\right|_{r=\varepsilon R} \approx \frac{a_{0}}{R} \frac{1}{\varepsilon|\ln (\varepsilon)|} .
$$

This shows explicitly how the cross-sectional slope of the (ideal) annular membrane at its inner rim becomes infinite in the pinned circular limit $(\varepsilon \rightarrow 0)$, and explains how the annular mode function is able to approach the complete circular mode function for all nonzero $r$ as the core shrinks to a point. In the limit, the mode solution is discontinuous at the origin. Of course, the linear theory no longer actually applies since equation (7) contradicts the assumption of small deflections as $\varepsilon \rightarrow 0$. However, the above analysis does serve to explain precisely the fundamental frequency result which was obtained in [2] as a limit within the linear formulation. 


\section{THE COLLARED CIRCULAR MEMBRANE}

The properties of the pinned circular membrane revealed above may seem rather counter-intuitive. The behaviour of a complete circular membrane with fixed perimeter, i.e. Dirichlet boundary condition

$$
\mathrm{u}(\mathrm{r})=0 \text { at } \mathrm{r}=\mathrm{R} \text {, }
$$

might in fact be expected to be more like a membrane with excluded core if the inner boundary was free, i.e. satisfied the Neumann boundary condition

$$
\partial \mathrm{u} / \partial \mathrm{r}=0 \text { at } \mathrm{r}=\varepsilon \mathrm{R} \text {. }
$$

This corresponds to an inner massless "collar" free to slide orthogonally to the plane of the undeflected annular membrane. We now show that the behaviour of the fundamental mode of this collared annular membrane indeed approaches that of the complete circular membrane as the collar radius $\varepsilon$ tends to zero. (For the fundamental mode of the complete circular membrane, it is not necessary to specify the condition $\partial u / \partial r=0$ at the centre, as it is a consequence of the standard solution.)

The modal function will be written here as

$$
\mathrm{u}_{0}(\mathrm{r})=\mathrm{a}_{0} \mathrm{~J}_{0}(\mathrm{Kr})+\mathrm{b}_{0} \mathrm{Y}_{0}(\mathrm{Kr})
$$

The characteristic equation following from the boundary conditions (8) and (9) is then

$$
\mathrm{J}_{0}(\mathrm{KR}) \mathrm{Y}_{1}(\varepsilon \mathrm{KR})-\mathrm{Y}_{0}(\mathrm{KR}) \mathrm{J}_{1}(\varepsilon \mathrm{KR})=0 .
$$

For small $\varepsilon$, a careful tracking of orders of $\varepsilon$ eventually leads to

$$
\begin{gathered}
K R=j_{0,1}+\mu, \\
\mu \approx \frac{\pi}{4} j_{0,1}{ }^{2} \frac{Y_{0}\left(j_{0,1}\right)}{J_{1}\left(j_{0,1}\right)} \varepsilon^{2} \approx 4.461409 \varepsilon^{2} .
\end{gathered}
$$


Thus

$$
\mathrm{KR} \rightarrow \mathrm{j}_{0,1} \quad \text { as } \varepsilon \rightarrow 0
$$

so, as anticipated, the fundamental frequency for the collared annular membrane approaches that of the complete circular membrane as the collar radius shrinks to zero, and does so fairly rapidly because of the 2 nd order smallness in (12b). In contrast to the pinned case of Section 2 above, the approximation $(12 a, b)$ to the exact solution to eq.(11) is also very good. For instance, even with $\varepsilon=0.1$, the approximation $(12 a, b)$ agrees with the exact solution to eq.(11) to almost 4 significant figures: $K R \approx 2.448$ (exact eq.(11)); 2.449 (eqs.(12).

Further analysis shows that, for the coefficients in the mode function (10),

$$
\mathrm{b}_{0} / \mathrm{a}_{0}=-\mathrm{J}_{0}(\mathrm{KR}) / \mathrm{Y}_{0}(\mathrm{KR}) \approx(\pi / 4) \mathrm{j}_{0,1}{ }^{2} \varepsilon^{2}
$$

and the product $\mathrm{b}_{0} \mathrm{Y}_{0}(\varepsilon \mathrm{KR})$ appearing in the mode function (10) at the inner (collar) boundary approaches zero like $-\left(\mathrm{a}_{0} / 2\right) \mathrm{j}_{0,1}{ }^{2} \varepsilon^{2}|\ln (\varepsilon)|$ as $\varepsilon \rightarrow 0$. Thus at the collar $\mathrm{u}_{0}(\varepsilon \mathrm{KR}) \rightarrow \mathrm{a}_{0}$ as $\varepsilon \rightarrow 0$. For any $\mathrm{r}$, in $(10) \mathrm{u}_{0}(\mathrm{r}) \rightarrow \mathrm{a}_{0} \mathrm{~J}_{0}\left(\mathrm{j}_{0,1} \mathrm{r} / \mathrm{R}\right)$ as $\varepsilon \rightarrow 0$, i.e. the mode function tends to the complete circle mode function as the collar shrinks to zero. Unlike the pinned membrane case, the limiting function is continuous at the origin, with the expected value $\mathrm{a}_{0}$.

The slope $\mathrm{du}_{0} / \mathrm{dr}$ at $\mathrm{r}=\varepsilon \mathrm{R}$ can be shown to be less than order $\varepsilon$ for $\varepsilon$ small. Thus the slope tends to zero as the collar shrinks, and in the limit the centre of the membrane is an extremum as for the complete membrane. It is therefore this nearlyclosed free-fixed annular membrane which has the property that its fundamental mode is everywhere near that of a complete circular membrane. 


\section{DISCUSSION}

Whilst it is obvious that central pinning of a circular membrane will not affect those modes having a nodal line passing through the origin (i.e. $n \geq 1$ in eq.(1)), it is perhaps unexpected that pinning will not affect the fundamental mode frequency, as described by Wang [2] and elaborated upon in Section 2 above. Indeed, it will not affect any mode since this conclusion evidently holds for the higher circularlysymmetric modes by replacing the lowest solution of equation (2) by the higher solutions and the first zero $\mathrm{j}_{0,1}$ of $\mathrm{J}_{0}$ by its higher order zeros $\mathrm{j}_{0, \mathrm{~s}}$.

This phenomenon appears to be related to the "infinite capacity for pliancy" of an ideal membrane in relation to point effects. As discussed and diagrammed by Morse [5, p.176], unlike the case of the one-dimensional stretched string, a finite force acting over an infinitesimal region of a tensioned ideal membrane produces an indefinitely large deflection. Thus the ideal membrane is able to sustain indefinitely large changes, and hence can adapt itself to accommodate the narrow well-like crosssectional modal shapes with high gradients near the origin, as found at the end of Section 2 above. There also appears to be a similarity with classical billiards containing a circular obstacle whose radius shrinks to zero so that it becomes a point scatterer. As noted by Seba [6], since the trajectories which hit the resulting scattering point are of measure zero, the system does not "feel" the point scatter.

It should be stressed that all the preceding mathematical expositions assume an ideal membrane satisfying the linear two-dimensional constant-speed wave equation with simple boundary conditions. Whilst we are not in a position to perform experimental work, we would expect that for a real circular membrane pinned at the centre (finite but very small excluded central core), the lower angle-dependent modal 
frequencies and cross-sectional shapes might be reasonably close to those of the complete circular membrane (whose modes have the origin on a nodal line). However, the frequencies, and especially the cross-sectional mode shapes, of the angleindependent modes of a real pinned membrane would be expected to be quite different from those of the unpinned system with an anti-node at the origin, due to the finite stiffness of the membrane which would mitigate against the mathematical "pliancy" mentioned above. Rayleigh [1] deduced that fixing more than one point of an (ideal) membrane does not alter the characteristic frequency. According to the preceding statement, we would expect that in these instances the real behaviour would be even less like the mathematical result.

Rayleigh also deduced that the results should hold for ideal membranes of any shape. Wang [2] dealt with regular polygonal membranes with circular core, and confirmed this numerically for the hexagon as the core radius shrank to zero.

Finally, we may conjecture that, because pinning is a local phenomenon, the shape of the small excluded core should not affect the above results. For instance, the calculations of [2] might be repeated for a small central polygonal core similar to the outer shape, with point matching on inner as well as outer boundary. 


\section{REFERENCES}

1. J.W.S. RAYLEIGH 1945 The Theory of Sound, Volume I, second edition. New York: Dover.

2. C.Y. WANG 1998 Journal of Sound and Vibration 215, 195-199. On the polygonal membrane with a circular core.

3. M. ABRAMOWITZ and I.A. STEGUN (editors) 1972 Handbook of Mathematical Functions, Chapter 9: F.W.J. OLVER, Bessel functions of integer order. New York: Dover.

4. A. JEFFREY 1995 Handbook of Mathematical Formulas and Integrals. San Diego: Academic Press.

5. P.M. MORSE 1983 Vibration and Sound, second edition, 1948. American Institute of Physics reprint.

6. P. SEBA 1990 Physical Review Letters 64, 1855-1858. Wave chaos in singular quantum billiard. 